\title{
WIRELESS BODY AREA NETWORKS: SECURITY AND PRIVACY ISSUES
}

\author{
Romina Muka $^{{ }^{*}}$, Kreshnik Vukatana $^{1}$, Kozeta Sevrani $^{1}$ \\ ${ }^{1 *}$ University of Tirana, Faculty of Economy, Tirana, Albania; \\ ${ }^{2 *}$ Norwegian University of Science and Technology, Faculty of Information Technology and Electrical Engineering, \\ Trondheim, Norway; \\ *Corresponding Author Romina Muka,e-mail: rominamuka@gmail.com; romina.muka@unitir.edu.al; \\ k.vukatana@gmail.com; kozeta.sevrani@unitir.edu.al;
}

Received June 2020; Accepted July 2020; Published August 2020;

DOI: https://doi.org/10.31407/ijees10.315

\begin{abstract}
The future of ubiquitous health technologies looks promising because of the great potential that Wireless Body Area Networks (WBANs) have. Latest advances in wireless communication technologies and sensors have emerged as a key technology to provide real-time health monitoring of a patient, collecting data of a patient's vital body parameters continuously and also diagnosing many life-threatening diseases. The main purpose of WBAN devices is to collect sensitive information, so providing strict security mechanisms is considered to be essential, in order to prevent situations where data may be lost or modified for malicious reasons. As a result, designers of WBANs should be more focused on creating secure systems, that minimize the possibilities of a misfunction or data corruption from a malicious program. This paper presents an analysis of WBANs related to security and privacy issues. Furthermore, it gives a brief insight into the appropriate security mechanisms to follow. Finally, it is highlighted how necessary it is to implement security solutions, in order to enhance the development of such systems in general.
\end{abstract}

Keywords: WBAN, e-Health, Security, Solutions, Privacy 